\title{
Survival and Safety of Patients with Inoperable Pancreatic Cancer in Clinical Trials That Haven't Demonstrated Increase of Overall Survival
}

\author{
Daniela Bajzath, Maxim Belotserkovskiy*, Kamen Doxev and Maxim Kosov \\ PSI CRO AG, Zug Switzerland
}

*Corresponding author: Maxim Belotserkovskiy, PSI CRO AG (Zug, Switzerland), PSI CRO Deutschland GmbH, Behringstr - 7, 82152

Planegg/Munich, Germany

\begin{abstract}
ARTICLE INFO
Received: 幽 October 24, 2019

Published: 幽 November 04, 2019

Citation: Daniela Bajzath, Maxim Belotserkovskiy, Kamen Doxev, Maxim Kosov.

\section{ABSTRACT}

Survival and safety of patients with inoperable pancreatic cancer in clinical trials that haven't demonstrated increase of overall survival are reviewed from the point of potential benefits for patients and appropriateness of patients' participation. A retrospective analysis summarizes safety data from 4 completed clinical trials with an aim to give an overview of Serious Adverse Events occurrence in study subjects, as well as to detail the events according to System Organ Class.
\end{abstract} Survival and Safety of Patients with Inoperable Pancreatic Cancer in Clinical Trials That Haven't Demonstrated Increase of Overall Survival. Biomed J Sci \& Tech Res 22(3)-2019. BJSTR. MS.ID.003758.
Keywords: Overall Survival; Inoperable Pancreatic Cancer; Clinical Trials; Safety

Abbreviations: ECOG: Eastern Cooperative Oncology Group; SAE: Serious Adverse Event; SOC: System Organ Class; SAR: Serious Adverse Reaction

\section{Introduction}

All randomized clinical trials in oncology, where the use of new medicines results in an increase in patient life expectancy, improvement of their quality of life, or at least improvement in progression-free survival, always have a broad and positive impact on the industry as they chart a new, more effective and coherent path towards better strategies in treatment of these patients. When planning a trial, a sponsor always strives for the best for patients. At the same time, we all know that statistically the majority of clinical trials (actually up to 85\%), including trials in advanced-stage and metastatic cancer (Stages III and IV), do not achieve their set goals (end-points) [1]. This always raises concerns in terms of potential benefits for patients and appropriateness of patients' participation.

\section{Materials and Methods}

We analysed the results of 4 completed clinical trials in patients with inoperable pancreatic cancer who at the enrolment stage had ECOG performance status of 0 - 1 and an estimated survival of at least 12 weeks. By the time of enrolment, the subjects of the trials had previously progressed after the standard first-line chemotherapy, which included Gemcitabine as a monotherapy or in a standard combination. All 4 trials were randomized, double-blinded, Phase
III studies, with a total of 1661 patients enrolled [2]. None of the studies, unfortunately, achieved their primary endpoints (overall survival), but neither were they stopped due to futility after an interim analysis. That is, all the patients kept participating in the study till treatment intolerance, or pancreatic cancer progression, or death. They could also have been excluded from the trial once the particular number of events (deaths) was reported in accordance to the Statistical Analysis Plan of the study.

\section{Analysis of Survival}

According to literature data, patients diagnosed with late stage inoperable metastatic pancreatic cancer have an overall survival of approximately 106 days (3.5 months) without standard treatment. Whereas with standard chemotherapy treatment, the survival increases to 182 days (6 months) [3-5]. In the 4 analysed clinical trials the average overall survival was found to be 204 days (221 days in the study drug arm and 188 days in the comparator arm). It is important to note that although the experimental treatment in the analysed trials did not prove to be effective for the fight against cancer, it did not reduce the overall survival of the participating patients either. The difference was not significant (Figure 1). 


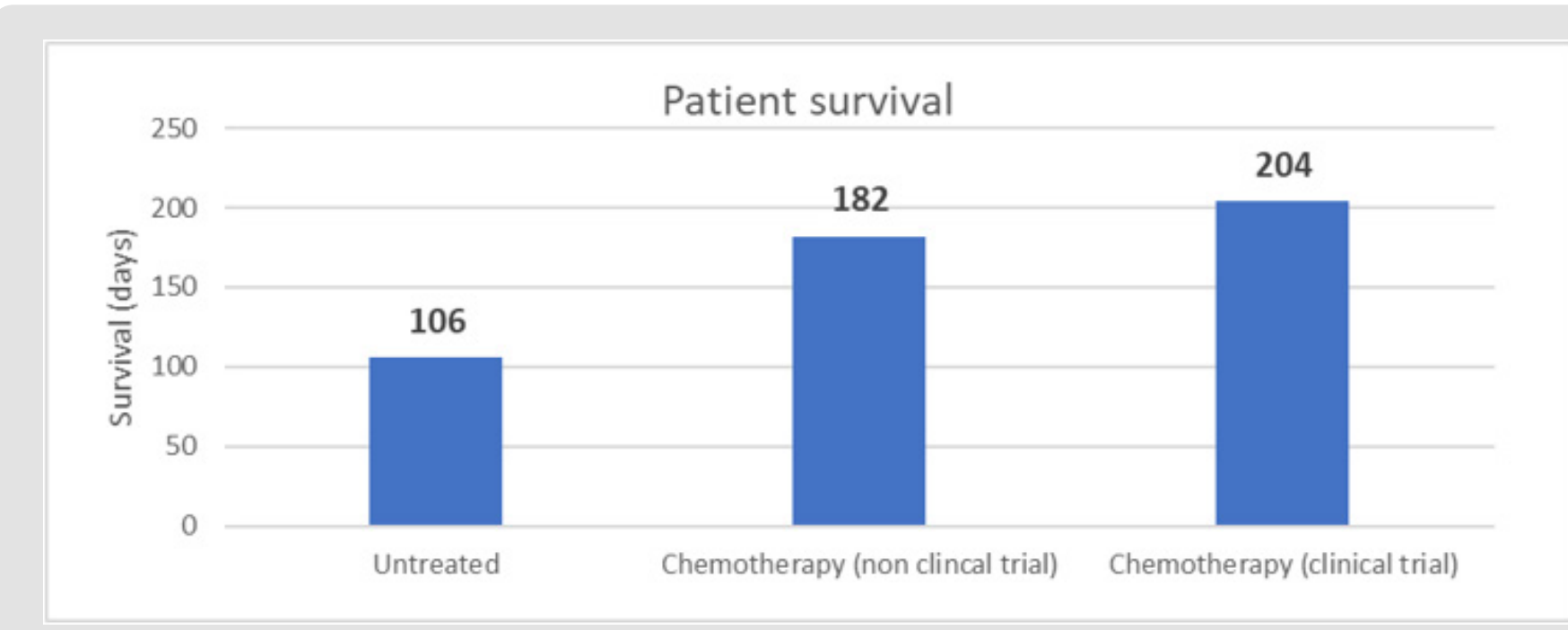

Figure 1.

\section{Analysis of SAES}

To evaluate the impact of study therapy on the safety of the study subjects, we analysed the structure of serious adverse events (SAE). In total, 1080 SAEs were reported in 684 patients within all 4 studies. As many as 180 SAEs reported in 132 patients were assessed as related to the study treatment by investigators. As all 4 trials were double-blinded, the investigators did not know the exact treatment assignments and the potential cause of these SAEs was either the study drug or comparator. During the studies, 153 patients died due to an SAE other than pancreatic cancer progression, and another 213 patients died from of pancreatic cancer progression (Table 1). The predominant seriousness criterion was hospitalization for a total of 752 SAEs (69.6\%). The next most common seriousness criterion was death for 178 SAEs (16.5\%). 23 SAEs $(2.1 \%)$ were classified as life-threatening and 4 cases $(0.4 \%)$ as development of disability. The analysis of the distribution of SAEs by System Organ Class (SOC) showed that 23\% of all reported adverse events fell within gastrointestinal disorders class with abdominal pain, vomiting, nausea, diarrhoea, constipation and gas- trointestinal haemorrhage as the most common events. The second frequent group was coded as general disorders that accounted for $16 \%$ of SAEs with pyrexia, disease progression, asthenia and general physical health deterioration at the top of the list. The third leading group referred to infections that made up 14\% of all SAEs with pneumonia, cellulitis, sepsis and febrile neutropenia being the most frequent conditions (Figure 2). Among the study drug related SAEs (180 cases), the leading causes followed the same pattern of SOCs representation. Nevertheless, these groups positioned differently on the list of the most common causes: the group of general disorders achieved the highest rate of $25 \%$ of SAEs with pyrexia and general physical health deterioration as the most frequently reported; gastrointestinal disorders went number two and reached $18 \%$ with vomiting, diarrhoea and nausea as the leading causes; and infections obtained $14 \%$ with prevailing diagnoses of febrile neutropenia, pneumonia and sepsis (Figure 3). The top five underlying causes of death were gastrointestinal disorders (20\%), general disorders (18\%), infections (11\%), hepatobiliary disorders (9\%) and respiratory disorders (9\%) (Figure 4).

\section{Study drug related SAE distribution by System Organ Class}

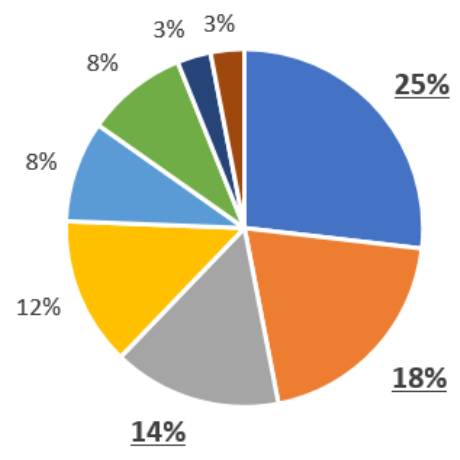

- General disorders

- Gastrointestinal disorders

- Infections

- Metabolism disorders

- Respiratory disorders

- Blood disorders

- Investigations

- Vascular disorders

Figure 2. 
Table 1.

\begin{tabular}{|c|c|c|c|c|c|}
\hline & Study 1 & Study 2 & Study 3 & Study 4 & TOTAL \\
\hline Number of patients & 823 & 400 & 153 & 285 & 1661 \\
\hline Number of SAEs & 429 & 469 & 138 & 44 & 1080 \\
\hline Number of patients with SAEs & 383 & 207 & 56 & 38 & 684 \\
\hline Number of SARs (drug-related SAEs) & 160 & 19 & 1 & 0 & 180 \\
\hline Number of patients with SARs & 117 & 14 & 1 & 0 & 132 \\
\hline Number of patients died & 32 & 72 & 38 & 11 & 153 \\
\hline
\end{tabular}

- Gastrointestinal disorders
- General disorders
- Infections
-
- Mespiratory disorders
- Metabolism disorders
- Vascular disorders

Figure 3.

\title{
Death causes distribution by System Organ Class
}

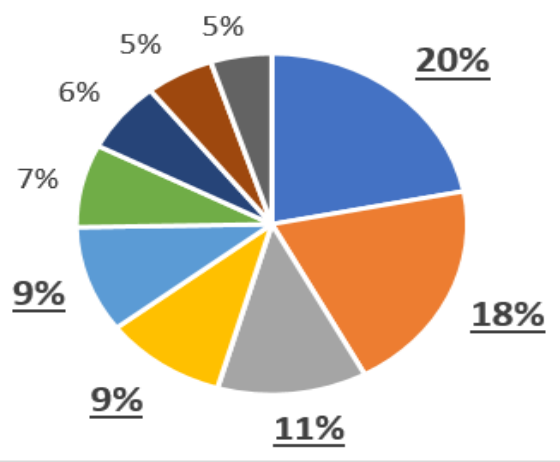

\author{
- Gastrointestinal disorders \\ - General disorders \\ - Infections \\ - Hepatobiliary disorders \\ - Respiratory disorders \\ - Cardiac disorders \\ - Tumors (benign and malignant)
}

Figure 4 .

Thus, analysis of SAEs showed the predominance of progressing malignancy or well-known side effects of chemo or immunotherapies. This distribution was well described in literature and, thus, is expected [6]. At the same time, it is important to note that infections comprised a significant share of SAEs, including those serious adverse drug reactions that were assessed by the investigators as study drug related, and they were considered the cause of death. Therefore, it could be recommended to maximize awareness and readiness to react as early as possible in order not to overlook development of diseases and to provide effective treatment immediately. It is also necessary to carefully evaluate patient's ability to maintain and tolerate the treatment and, in case of signs of the development of such previously mentioned general disorders, to take all required actions and measures for correction of these disorders up to and including termination of treatment.

\section{Conclusion}

Participation of patients in clinical trials, even in cases when experimental therapy did not prove its efficacy, doesn't result in decrease of patient's overall survival and, thus, can also be recommended considering high standards of diagnostics and care in clinical trials. Analysis of 4 clinical trials in patients with inoperable pancreatic cancer showed an average overall survival of 204 days, which is not lower than the survival rate with standard 
of care chemotherapy treatment, although none of the analysed studies met its primary endpoints, neither were experimental study treatments marketed later on. Gastrointestinal disorders, general disorders and infections were the most frequently reported serious adverse events, as well as the most frequent causes of death irrespective of the treatment arm - study therapy or comparator. During clinical trials in pancreatic cancer it is critically important to pay additional attention to the initial and even minimal clinical and laboratory signs and symptoms of infection to ensure the highest possible level of active monitoring and immediate treatment of detected disorders.

\section{References}

1. https://www.centerwatch.com/cwweekly/2018/02/05/new-mitstudy-puts-clinical-research-success-rate-14-percent/

\section{ISSN: 2574-1241}

DOI: 10.26717/BJSTR.2019.22.003758

Maxim Belotserkovskiy. Biomed J Sci \& Tech Res

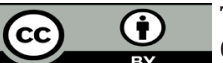

This work is licensed under Creative Commons Attribution 4.0 License

Submission Link: https://biomedres.us/submit-manuscript.php
2. (2019) Pancreatic Cancer. American Cancer Society.

3. B Glimelius (1996) Chemotherapy improves survival and quality of life in advanced pancreatic and. Annals of Oncology 7(6): 593-600.

4. DN Lakdawalla (2017) Predicting Real-World Effectiveness of Cancer Therapies Using Overall Survival and Progression-Free Survival from Clinical Trials: Empirical Evidence for the ASCO Value Framework. Value Health 20(7): 866-875.

5. RR Sarkar, R M (2016) Pancreatic cancer: Survival in clinical trials versus the real world. Journal of Clinical Oncology 34(4 suppl): 216

6. M Tong, J W (2019) Efficacy and safety of gemcitabine plus antiangiogenesis therapy for advanced pancreatic cancer: A systematic review and meta-analysis of clinical randomized phase III trials. Journal of cancer 10(4): 968-978.

BIOMEDICAL
RESEARCHES $\quad \begin{aligned} & \text { Assets of Publishing with us } \\ & \text { - Global archiving of articles }\end{aligned}$

